\title{
Minimally invasive versus open fusion for Grade I degenerative lumbar spondylolisthesis: analysis of the Quality Outcomes Database
}

\author{
Praveen V. Mummaneni, MD, ${ }^{1}$ Erica F. Bisson, MD, MPH, ${ }^{2}$ Panagiotis Kerezoudis, MD, ${ }^{3}$ \\ Steven Glassman, MD, ${ }^{4}$ Kevin Foley, MD, ${ }^{5}$ Jonathan R. Slotkin, MD, ${ }^{6}$ Eric Potts, MD, ${ }^{7}$ \\ Mark Shaffrey, MD, ${ }^{8}$ Christopher I. Shaffrey, MD, ${ }^{8}$ Domagoj Coric, MD, ${ }^{9}$ John Knightly, MD, ${ }^{10}$ \\ Paul Park, MD, ${ }^{11}$ Kai-Ming Fu, MD, PhD, ${ }^{12}$ Clinton J. Devin, MD, ${ }^{13}$ Silky Chotai, MD,13 \\ Andrew K. Chan, MD, ${ }^{1}$ Michael Virk, MD, PhD, ${ }^{1}$ Anthony L. Asher, MD, ${ }^{9}$ and Mohamad Bydon, MD ${ }^{3}$
}

\begin{abstract}
1Department of Neurological Surgery, University of California, San Francisco, California; 'Department of Neurological Surgery, University of Utah, Salt Lake City, Utah; ${ }^{3}$ Department of Neurologic Surgery, Mayo Clinic, Rochester, Minnesota; ${ }^{4}$ Norton Leatherman Spine Center, Louisville, Kentucky; ${ }^{5}$ Department of Neurosurgery, University of Tennessee, Memphis, Tennessee; ${ }^{6}$ Geisinger Health System, Danville, Pennsylvania; ${ }^{7}$ Department of Neurological Surgery, Indiana University, Goodman Campbell Brain and Spine, Indianapolis, Indiana; ${ }^{8}$ Department of Neurological Surgery, University of Virginia, Charlottesville, Virginia; ${ }^{9}$ Neuroscience Institute, Carolinas Healthcare System and Carolina Neurosurgery \& Spine Associates, Charlotte, North Carolina; ${ }^{10}$ Atlantic Neurosurgical Specialists, Morristown, New Jersey; ${ }^{11}$ Department of Neurologic Surgery, University of Michigan, Ann Arbor, Michigan; ${ }^{12}$ Department of Neurological Surgery, Weill Cornell Medical College, New York, New York; and ${ }^{13}$ Department of Orthopedic Surgery, Vanderbilt University, Nashville, Tennessee
\end{abstract}

OBJECTIVE Lumbar spondylolisthesis is a degenerative condition that can be surgically treated with either open or minimally invasive decompression and instrumented fusion. Minimally invasive surgery (MIS) approaches may shorten recovery, reduce blood loss, and minimize soft-tissue damage with resultant reduced postoperative pain and disability. METHODS The authors queried the national, multicenter Quality Outcomes Database (QOD) registry for patients undergoing posterior lumbar fusion between July 2014 and December 2015 for Grade I degenerative spondylolisthesis. The authors recorded baseline and 12-month patient-reported outcomes (PROs), including Oswestry Disability Index (ODI), EQ-5D, numeric rating scale (NRS)-back pain (NRS-BP), NRS-leg pain (NRS-LP), and satisfaction (North American Spine Society satisfaction questionnaire). Multivariable regression models were fitted for hospital length of stay (LOS), 12-month PROs, and 90-day return to work, after adjusting for an array of preoperative and surgical variables. RESULTS A total of 345 patients (open surgery, $n=254$; MIS, $n=91$ ) from 11 participating sites were identified in the QOD. The follow-up rate at 12 months was $84 \%$ (83.5\% [open surgery]; 85\% [MIS]). Overall, baseline patient demographics, comorbidities, and clinical characteristics were similarly distributed between the cohorts. Two hundred fifty seven patients underwent 1-level fusion (open surgery, $n=181$; MIS, $n=76$ ), and 88 patients underwent 2-level fusion (open surgery, $n=73$; MIS, $n=15$ ). Patients in both groups reported significant improvement in all primary outcomes (all $p<0.001$ ). MIS was associated with a significantly lower mean intraoperative estimated blood loss and slightly longer operative times in both 1- and 2-level fusion subgroups. Although the LOS was shorter for MIS 1-level cases, this was not significantly different. No difference was detected with regard to the 12-month PROs between the 1-level MIS versus the 1-level open surgical groups. However, change in functional outcome scores for patients undergoing 2-level fusion was notably larger in the MIS cohort for ODI (-27 vs $-16, p=0.1)$, EQ-5D (0.27 vs $0.15, p=0.08)$, and NRS-BP (-3.5 vs $-2.7, p=0.41)$; statistical significance was shown only for changes in NRS-LP scores $(-4.9$ vs $-2.8, p=0.02)$. On riskadjusted analysis for 1-level fusion, open versus minimally invasive approach was not significant for 12-month PROs, LOS, and 90-day return to work.

CONCLUSIONS Significant improvement was found in terms of all functional outcomes in patients undergoing open or MIS fusion for lumbar spondylolisthesis. No difference was detected between the 2 techniques for 1-level fusion in terms 
of patient-reported outcomes, LOS, and 90-day return to work. However, patients undergoing 2-level MIS fusion reported significantly better improvement in NRS-LP at 12 months than patients undergoing 2-level open surgery. Longer followup is needed to provide further insight into the comparative effectiveness of the 2 procedures.

https://thejns.org/doi/abs/10.3171/2017.5.FOCUS17188

KEY WORDS lumbar; spondylolisthesis; open surgery; minimally invasive surgery; Quality Outcomes Database

$\mathrm{D}$ EGENERATIVE lumbar spondylolisthesis is a chronic condition characterized by the slippage of a vertebra relative to the segment below. It is considered an important cause of chronic low-back and radicular pain, with an estimated prevalence of $12 \%$ in the adult United States population. ${ }^{1,11}$ Although generally a benign condition, spondylolisthesis may progress with time in terms of both clinical symptoms and increase in anterolisthesis. ${ }^{21}$ Surgery, offered to patients in whom optimal medical management has failed, typically entails either decompression alone or decompression with fusion.

Open approaches are considered the gold standard when instrumented fusion is used, with reliable improvement in improving patients' function and quality of life. ${ }^{15}$ However, an open approach might further destabilize supporting structures such as the facet joints and ligamentous structures..${ }^{15}$ Minimally invasive surgery (MIS), including anterior, lateral, or posterior lumbar interbody fusion, has attracted increasing attention within the last decade (Fig. 1). Modern MIS techniques allow for visualization of the screw entry points while inspecting the instrumentation and neural structures. ${ }^{5,18}$ Proponents of MIS fusion suggest that it leads to reduced blood loss, shorter recovery, and less postoperative pain, while minimizing soft-tissue dissection and maintaining the structural integrity of the paraspinal muscles.

Previous studies have shown mixed results with regard to the efficacy of open versus minimally invasive fusion for spondylolisthesis. ${ }^{2,9,10,13,19,20,22-24}$ Moreover, these studies are limited by their single-institution nature, thereby reflecting the experience of relatively few (1-4) surgeons. In light of the limitations in the current literature, we conducted a retrospective analysis of prospectively collected data in a multiinstitutional spine registry that represents a real-world spinal practice comprising academic and private institutions.

\section{Methods \\ Data Source}

We queried the Quality Outcomes Database (QOD) registry for patients who underwent spinal fusion for degenerative spondylolisthesis between July 2014 and December 2015. The QOD is a prospective registry into which patients have been enrolled since 2012. It is designed to evaluate risk-adjusted expected morbidity and 12-month outcomes with the aim of improving efficiency and quality of care for the most commonly performed spinal surgical procedures. ${ }^{16}$ As of March 2016, more than 23,000 patients across 80 participating sites nationwide have been enrolled in the Spine Surgery QOD. ${ }^{16}$ Of these, 11 sites proposed a focused project to determine the impact of fusion on patient-reported outcomes (PROs). Preoperative radiographs, standing or dynamic, were evaluated by surgeons at the participating site to confirm the diagnosis of Grade I spondylolisthesis. All surgical variables, including laminectomy performed, fusion performed, and number of levels of fusion or laminectomy, MIS versus open surgery, were audited for all eligible patients. Patients who underwent elective lumbar spine surgery for Grade I spondylolisthesis (according to the Meyerding classification ${ }^{17}$ (via a posterior-only approach were included in the analysis. In addition to QOD exclusion criteria, ${ }^{16}$ patients with Grade II or higher spondylolisthesis other than a posterioronly approach, more than 2 levels of fusion, and cortical screw fixation were excluded. Minimally invasive spinal fusion was defined as the utilization of percutaneous or tubular screw fixation, with or without intervertebral body graft placement.

\section{Outcomes of Interest}

The primary outcome measures were assessed at 12 months using validated questionnaires and included the Oswestry Disability Index (ODI) ${ }^{8}$ numeric rating scale (NRS) for back pain (NRS-BP) and leg pain (NRS-LP), ${ }^{14}$ preference-based health status (EQ-5D), ${ }^{7}$ and North American Spine Society (NASS) patient satisfaction score. ${ }^{4}$ Patients responding "yes" to Question 1 or 2 of the NASS patient satisfaction questionnaire (Question 1: "The treatment met my expectations." Question 2: "I did not improve as much as I had hoped, but I would undergo the same treatment for the same outcome.") were considered satisfied. Secondary outcome measures included intraoperative blood loss, operative time, hospital length of stay (LOS) as well as 90 -day readmission, 90 -day return to work, and return to the operating room within 1 year.

\section{Covariates}

We recorded the following information: 1) demographics: age, sex, body mass index (BMI), ethnicity, insurance, education level, employment, and workers' compensation; 2) comorbidities: smoking, diabetes, anxiety, osteoporosis, depression, and American Society of Anesthesiologists (ASA) Physical Classification Status; 3) clinical characteristics: symptom duration, dominant symptom, ambulation, and presence of motor deficit; 4) baseline PRO scores: ODI, EQ-5D, NRS-LP, NRS-BP; and 4) surgical variables: number of levels of fusion.

\section{Statistical Analysis}

Separate analyses were conducted for patients undergoing 1- or 2-level fusion. Descriptive statistics (means and standard deviations for continuous variables; frequencies and proportions for categorical variables) were used for available patient demographic and comorbidity information 

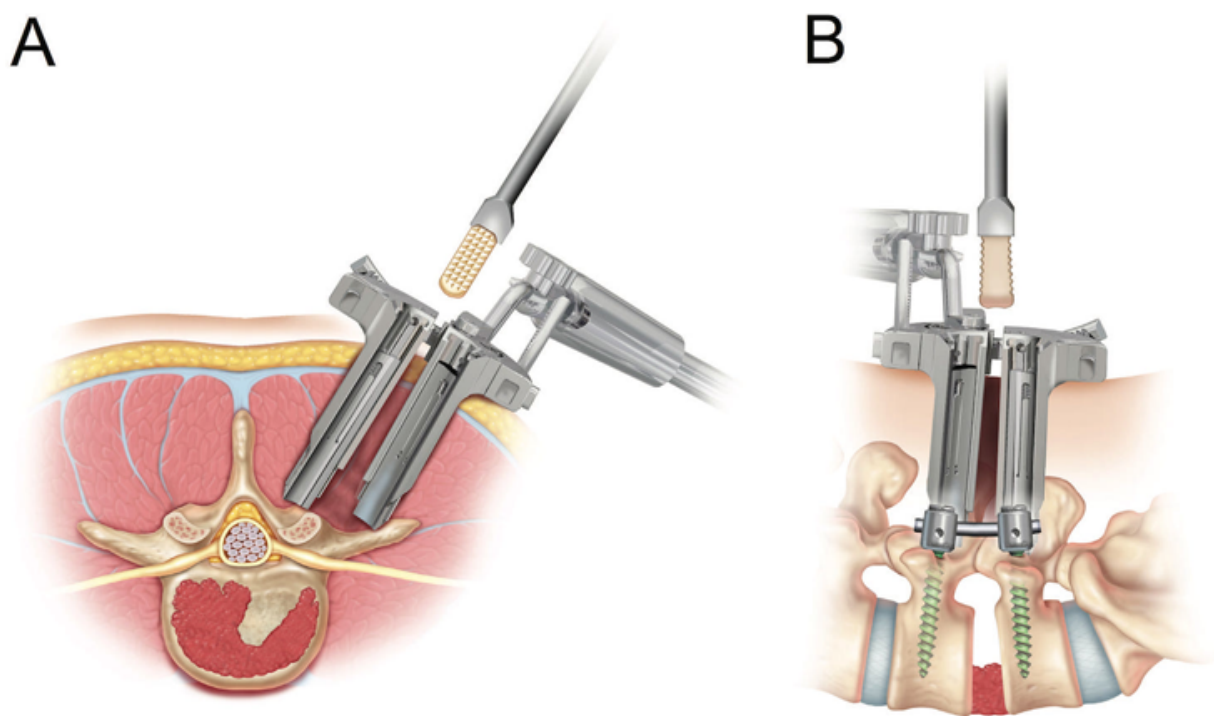

FIG. 1. Illustrations of axial (A) and lateral (B) views depicting placement of the interbody cage through the expandable tube during the MIS TLIF. Reprinted with permission from Dhall et al: J Neurosurg Spine 9:560-565, 2008.

as well as baseline symptoms, diagnosis, and PRO scores. Between the 2 cohorts, continuous variables were compared using an unpaired Student t-test, whereas categorical variables were compared using Pearson's chi-square and Fisher's exact tests. We also calculated quality-adjusted life days for the 1st year after surgery using the EQ-5D scores at baseline and at 3 and 12 months. Multivariable proportional odds ordinal regression models were fitted for ODI, EQ-5D, NRS-BP, NRS-LP, NASS satisfaction, and LOS after controlling for the covariates of interest. Due to the limited sample size, multivariable analysis was performed only for patients undergoing 1-level fusion. Missing values in the data were imputed using the missForest $\mathrm{R}$ package, a nonparametric imputation method based on the random forest algorithm (http://adsabs.harvard.edu/abs/2015ascl. soft05011S). The missForest algorithm can handle highdimensional data sets with mixed-type variables, including continuous, categorical, and count data; nonlinear relationships; and complex interactions. The analysis was performed using R 3.1.2 (R: A language and environment for statistical computing. R Foundation for Statistical Computing, Vienna, Austria. URL https://www.R-project.org) and "rms" package. Probability values were 2-tailed and were considered significant if $<0.05$.

\section{Results}

\section{Patient Demographics and Comorbidities}

We identified a total of 402 patients from 11 centers in the QOD registry who underwent spinal fusion for spondylolisthesis. After excluding patients with Grade II spondylolisthesis $(\mathrm{n}=7)$, patients who underwent an approach other than a posterior-only approach $(n=42)$, more than 2 levels of fusion $(n=4)$, and cortical screw fixation $(n=4)$, our final cohort consisted of 345 patients (open surgery, $\mathrm{n}=254$; MIS, $\mathrm{n}=91$ ). Two hundred fifty-seven patients underwent 1-level fusion (open surgery, $\mathrm{n}=181$; MIS, $\mathrm{n}=$
76), and 88 patients underwent 2-level fusion (open surgery, $n=73$; MIS, $n=15$ ). The follow-up rate at 12 months was $84 \%$ (open surgery, $\mathrm{n}=83.5 \%$; MIS, $\mathrm{n}=85 \%$ ).

Overall, baseline demographics and comorbidities were similarly distributed between the 2 groups (Table 1 ). The mean age was 61 years (1-level: 61 years [open surgery] vs 60 years [MIS], $\mathrm{p}=0.43$; 2-level: 63 years [open surgery] vs 61 years [MIS], $\mathrm{p}=0.58$ ), and the majority were females (1-level: $64 \%$ [open surgery] vs $55 \%$ [MIS], $\mathrm{p}=0.27$; 2-level: $53 \%$ [open surgery] vs $73 \%$ [MIS], $\mathrm{p}=$ 0.26 ). Patients in the open group were more likely to be of Hispanic ethnicity (1-level: 24\% vs 3\%, p < 0.001; 2-level: $18 \%$ vs $0 \%, \mathrm{p}=0.22$ ). No differences were observed in either subgroup with regard to BMI, smoking status, coronary artery disease, diabetes, and ASA class. Variables related to socioeconomic status (education level, employment activity and insurance status) were also similar between the 2 cohorts.

\section{Baseline Clinical Characteristics}

Baseline symptoms, diagnoses, and PROs are presented in Table 2. The majority of patients in both groups had a symptom duration between 3 and 12 months $(p=0.33$ for 1 level; $p>0.99$ for 2 levels and rated back- and legrelated symptoms to be equally problematic $(p=0.07$ for 1 level; $p=0.54$ for 2 levels). Moreover, no difference was found with regard to the presence of motor deficit $(\mathrm{p}=$ 0.84 for 1 level; $p=0.75$ for 2 levels) or ambulation ( $p=$ 0.26 for 1 level; $p=0.74$ for 2 levels). In terms of baseline PRO scores, patients in the 1-level subgroup undergoing open surgery presented with a baseline severity of disease burden that was comparable to patients undergoing MIS, including mean ODI (45.1 vs 48.1, $\mathrm{p}=0.18$ ), EQ-5D score (0.54 vs $0.53, p=0.83)$, NRS-BP (7 vs $6.9, p=0.74$ ), and NRS-LP (6.7 vs $6.6, p=0.9)$. On the contrary, MIS patients in the 2-level subgroup presented with significantly more severe disability (ODI score 55.3 vs $42.1, \mathrm{p}=0.006$ ) 
TABLE 1. Patient demographics and comorbidities by approach

\begin{tabular}{|c|c|c|c|c|c|c|c|}
\hline \multirow[b]{2}{*}{ Variable } & \multirow[b]{2}{*}{ All $(n=345)$} & \multicolumn{3}{|c|}{ 1-Level Fusion } & \multicolumn{3}{|c|}{ 2-Level Fusion } \\
\hline & & Open $(n=181)$ & MIS $(n=76)$ & $p$ Value & Open $(n=73)$ & MIS $(N=15)$ & $p$ Value \\
\hline Age in yrs, mean (SD) & $61.0(10.7)$ & $60.9(11.0)$ & $59.8(10.5)$ & 0.43 & $62.5(10.5)$ & $61.1(7.87)$ & 0.58 \\
\hline Female sex, $\mathrm{n}(\%)$ & $207(60.0)$ & $115(63.5)$ & $42(55.3)$ & 0.27 & $39(53.4)$ & $11(73.3)$ & 0.26 \\
\hline BMI, mean (SD) & $31.2(6.93)$ & $31.1(7.27)$ & $31.0(6.91)$ & 0.93 & $31.4(6.33)$ & $31.8(6.14)$ & 0.82 \\
\hline Hispanic ethnicity, n (\%) & & & & $<0.001$ & & & 0.22 \\
\hline Yes & $58(16.8)$ & $43(23.8)$ & $2(2.63)$ & & $13(17.8)$ & $0(0.00)$ & \\
\hline Prefer not to answer & $9(2.61)$ & $5(2.76)$ & $1(1.32)$ & & $3(4.11)$ & $0(0.00)$ & \\
\hline Education, n (\%) & & & & 0.76 & & & 0.538 \\
\hline$<$ high school & $13(3.77)$ & $8(4.42)$ & $1(1.32)$ & & $4(5.48)$ & $0(0.00)$ & \\
\hline High school diploma & $126(36.5)$ & $64(35.4)$ & $31(40.8)$ & & $28(38.4)$ & $3(20.0)$ & \\
\hline 2-yr college degree & $74(21.4)$ & $38(21.0)$ & $15(19.7)$ & & $17(23.3)$ & $4(26.7)$ & \\
\hline 4-yr college degree & $74(21.4)$ & $39(21.5)$ & $14(18.4)$ & & $15(20.5)$ & $6(40.0)$ & \\
\hline Postcollege & $51(14.8)$ & $27(14.9)$ & $14(18.4)$ & & $8(11.0)$ & $2(13.3)$ & \\
\hline Prefer not to answer & $7(2.03)$ & $5(2.76)$ & $1(1.32)$ & & $1(1.37)$ & $0(0.00)$ & \\
\hline Smoking, n (\%) & $34(9.86)$ & $20(11.0)$ & $8(10.5)$ & $>0.99$ & $6(8.22)$ & $0(0.00)$ & 0.58 \\
\hline CAD, n (\%) & $40(11.6)$ & $24(13.3)$ & $5(6.58)$ & 0.18 & $9(12.3)$ & $2(13.3)$ & $>0.99$ \\
\hline Diabetes, n (\%) & $59(17.1)$ & $31(17.1)$ & $10(13.2)$ & 0.54 & $15(20.5)$ & $3(20.0)$ & $>0.99$ \\
\hline Anxiety, n (\%) & $60(17.4)$ & $33(18.2)$ & $10(13.2)$ & 0.42 & $15(20.5)$ & $2(13.3)$ & 0.73 \\
\hline Depression, n (\%) & $79(22.9)$ & $43(23.8)$ & $15(19.7)$ & 0.59 & $16(21.9)$ & $5(33.3)$ & 0.34 \\
\hline Osteoporosis, n (\%) & $22(6.38)$ & $11(6.08)$ & $6(7.89)$ & 0.80 & $3(4.11)$ & $2(13.3)$ & 0.2 \\
\hline ASA class, $n(\%)$ & & & & 0.28 & & & $>0.99$ \\
\hline$|/| \mid$ & $180(52.3)$ & $94(52.2)$ & $46(60.5)$ & & $33(45.2)$ & $7(46.7)$ & \\
\hline III/IV & $164(47.7)$ & $86(47.8)$ & $30(39.5)$ & & $40(54.8)$ & $8(53.3)$ & \\
\hline Employment, n (\%) & & & & 0.19 & & & 0.273 \\
\hline Employed \& currently working & $147(42.6)$ & $75(41.4)$ & $40(52.6)$ & & $28(38.4)$ & $4(26.7)$ & \\
\hline $\begin{array}{l}\text { Employed but not working (on short- } \\
\text { term disability or on leave) }\end{array}$ & $19(5.51)$ & $12(6.63)$ & $2(2.63)$ & & $3(4.11)$ & $2(13.3)$ & \\
\hline Unemployed & $179(51.9)$ & $94(51.9)$ & $34(44.7)$ & & $42(57.5)$ & $9(60.0)$ & \\
\hline Workers' Compensation, n (\%) & $6(1.72)$ & $3(1.69)$ & $1(1.32)$ & $>0.99$ & & & \\
\hline Insurance, n (\%) & & & & 0.05 & & & 0.89 \\
\hline Uninsured & $1(0.29)$ & $0(0.00)$ & $1(1.32)$ & & $0(0.00)$ & $0(0.00)$ & \\
\hline Medicare & $123(35.7)$ & $70(38.7)$ & $20(26.3)$ & & $26(35.6)$ & $7(46.7)$ & \\
\hline Medicaid & $18(5.22)$ & $11(6.08)$ & $3(3.95)$ & & $4(5.48)$ & $0(0.00)$ & \\
\hline VA/government & $12(3.48)$ & $8(4.42)$ & $1(1.32)$ & & $3(4.11)$ & $0(0.00)$ & \\
\hline Private & $191(55.4)$ & $92(50.8)$ & $51(67.1)$ & & $40(54.8$ & $8(53.3)$ & \\
\hline
\end{tabular}

$C A D=$ coronary artery disease $; \mathrm{VA}=$ Veterans Affairs.

Boldface type indicates statistical significance.

and worse quality of life (EQ-5D score 0.44 vs $0.56, \mathrm{p}$ $=0.049$ ). Pain scores were also higher but did not reach statistical significance (NRS-BP [7.2 vs 6.7, $p=0.5]$ and NRS-LP [7.1 vs $6.3, \mathrm{p}=0.26]$ ).

\section{Operative Characteristics}

Intraoperative variables are outlined in Table 3. The MIS approach was associated with lower mean blood loss ( 1 level, 143 vs $290 \mathrm{ml} ; 2$ levels, 220 vs $512 \mathrm{ml}$; both p < $0.001)$ and a slightly longer operative time (1 level, 212 vs 190 minutes [p $=0.07$ ]; 2 levels, 282 vs 226 minutes $[\mathrm{p}=0.1])$. (Hemi)laminectomy was performed in most cases of 1-level fusion (96\% [open surgery] vs 97\% [MIS], p > 0.99) and 2-level fusion (96\% [open surgery] vs $93 \%$
[MIS], $\mathrm{p}=0.53)$. Lastly, MIS patients were more likely to undergo an interbody graft placement $(98.7 \%$ [1 level] and $100 \%$ [2 levels]) compared with open surgery (84\% [1 level] and $85 \%$ [2 levels]).

\section{Postoperative Outcomes}

A summary of postoperative outcomes is presented in Table 4. The average hospital LOS was similar between the open surgery and MIS cohorts in the 1-level fusion group (3.4 vs 3.2 days, $p=0.53$ ) and the 2 -level fusion subgroup (3.9 vs 4 days, $\mathrm{p}=0.83$ ). Patients in both cohorts reported significant improvement in all primary outcomes (all p < 0.001) (Figs. 2 and 3). Discharge to home was the most common disposition for the open surgery 
TABLE 2. Baseline symptoms, diagnoses, and PRO scores by approach

\begin{tabular}{|c|c|c|c|c|c|c|c|}
\hline \multirow[b]{2}{*}{ Variable } & \multirow[b]{2}{*}{ All $(n=345)$} & \multicolumn{3}{|c|}{ 1-Level Fusion } & \multicolumn{3}{|c|}{ 2-Level Fusion } \\
\hline & & Open $(n=181)$ & MIS $(n=76)$ & p Value & Open $(n=73)$ & MIS (n = 15) & p Value \\
\hline Symptom duration, $\mathrm{n}(\%)$ & & & & 0.33 & & & $>0.99$ \\
\hline$<3$ mos & $5(1.45)$ & $5(2.76)$ & $0(0.00)$ & & $0(0.00)$ & $0(0.00)$ & \\
\hline $3-12 \mathrm{mos}$ & $327(94.8)$ & $167(92.3)$ & 74 (97.4) & & $71(97.3)$ & $15(100)$ & \\
\hline$>12$ mos & $13(3.77)$ & $9(4.97)$ & $2(2.63)$ & & $2(2.74)$ & $0(0.00)$ & \\
\hline Motor deficit present, n (\%) & $73(21.2)$ & $37(20.4)$ & $14(18.4)$ & 0.84 & $19(26.0)$ & $3(20.0)$ & 0.75 \\
\hline Ambulation, $\mathrm{n}(\%)$ & & & & 0.26 & & & 0.74 \\
\hline Independent & $306(88.7)$ & $166(91.7)$ & $66(86.8)$ & & $62(84.9)$ & $12(80.0)$ & \\
\hline w/ an assisting device & $36(10.4)$ & $13(7.18)$ & $10(13.2)$ & & $10(13.7)$ & $3(20.0)$ & \\
\hline Wheelchair bound (nonambulatory) & $3(0.87)$ & $2(1.10)$ & $0(0.00)$ & & $1(1.37)$ & $0(0.00)$ & \\
\hline Dominant symptom, $\mathrm{n}(\%)$ & & & & 0.07 & & & 0.54 \\
\hline Back & $127(36.8)$ & $70(38.7)$ & $20(26.3)$ & & $30(41.1)$ & $7(46.7)$ & \\
\hline Leg & $45(13.0)$ & $27(14.9)$ & $9(11.8)$ & & $9(12.3)$ & $0(0.00)$ & \\
\hline Back $=$ leg & $173(50.1)$ & $84(46.4)$ & $47(61.8)$ & & $34(46.6)$ & $8(53.3)$ & \\
\hline \multicolumn{8}{|l|}{ Baseline PROs, mean (SD) } \\
\hline ODI & $45.6(16.0)$ & $45.1(15.3)$ & $48.1(16.2)$ & 0.18 & $42.1(16.9)$ & $55.3(14.8)$ & 0.006 \\
\hline$E Q-5 D$ & $0.54(0.22)$ & $0.54(0.21)$ & $0.53(0.24)$ & 0.83 & $0.56(0.22)$ & $0.44(0.20)$ & 0.049 \\
\hline NRS-BP & $6.92(2.48)$ & $7.00(2.25)$ & $6.88(2.76)$ & 0.74 & $6.68(2.68)$ & $7.20(2.78)$ & 0.52 \\
\hline NRS-LP & $6.61(2.73)$ & $6.68(2.61)$ & $6.63(2.85)$ & 0.9 & $6.33(2.97)$ & $7.13(2.36)$ & 0.26 \\
\hline
\end{tabular}

Boldface type indicates statistical significance.

and MIS cohorts ( $p=0.76$ for 1 level; $p=0.91$ for 2 levels). No differences were detected in 90-day readmission and 12-month PROs between the cohorts.

PRO scores between 12 months and baseline were found to be comparable within the 1-level subgroup. On the contrary, in the 2-level subgroup there were notable differences between the MIS and open surgery cohorts in terms of ODI ( -27 vs $-16, \mathrm{p}=0.1)$, EQ-5D (0.27 vs 0.15 , $\mathrm{p}=0.08)$ and NRS-BP ( -3.5 vs $-2.7, \mathrm{p}=0.41)$; however, statistical significance was shown only for NRS-LP (-4.9 vs $-2.8, \mathrm{p}=0.02$ )

The mean quality-adjusted life days within the 1-level subgroup was 268 for open surgery and 270 for MIS ( $\mathrm{p}=$ 0.84 ), whereas within the 2-level subgroup they were 253 for open surgery and 262 for MIS $(p=0.45)$. Although not statistically significant, more patients in the MIS cohort returned to work within 90 days (1 level: 77\% [MIS] vs $61 \%$ [open surgery], $\mathrm{p}=0.18 ; 2$ levels: $100 \%$ [MIS] vs $63 \%$ [open surgery], $\mathrm{p}=0.27$ ). Return to the operating within 1 year for conditions related to the index procedure was very uncommon in our overall cohort (20 patients), with the most common reason being surgical site infection $(\mathrm{n}=$ $6)$, followed by adjacent-segment disease $(n=3)$ and hardware failure $(n=2)$.

\section{Multivariable Regression Analysis for the 1-Level Fusion Subgroup}

Multivariable proportional odds logistic regression analysis did not reveal any significant differences with regard to the outcomes of interest: 12-month ODI (OR 1.01, 95\% CI 0.59-1.72; $\mathrm{p}=0.96$ ), EQ-5D (OR 0.87, 95\% CI 0.52-1.48; $\mathrm{p}=0.61)$, NRS-LP (OR 0.69, 95\% CI 0.39-1.21; $\mathrm{p}=0.2)$, NRS-BP (OR 1.05, 95\% CI 0.62-1.78 p = 0.85), satisfaction (OR 1.52, 95\% CI 0.75-3.10, $\mathrm{p}=0.25)$, LOS (OR 0.80, 95\% CI 0.46-1.39; p = 0.43), and 90-day return to work (OR 1.46, 95\% CI 0.21-10.3; $\mathrm{p}=0.70)$ (Table 5).

\section{Discussion}

The utilization of MIS approaches to treat degenera-

TABLE 3. Summary of intraoperative variables

\begin{tabular}{lcccccccc}
\hline & & \multicolumn{3}{c}{ 1-Level Fusion } & & \multicolumn{3}{c}{ 2-Level Fusion } \\
\multicolumn{1}{c}{ Variable } & All $(\mathrm{n}=345)$ & Open $(\mathrm{n}=181)$ & MIS $(\mathrm{n}=76)$ & $\mathrm{p}$ Value & & Open $(\mathrm{n}=73)$ & MIS $(\mathrm{n}=15)$ & $\mathrm{p}$ Value \\
\hline EBL in ml, mean (SD) & $303(296)$ & $290(219)$ & $143(116)$ & $<0.001$ & & $512(452)$ & $220(152)$ & $<0.001$ \\
\hline Op time in mins, mean (SD) & $206(81.2)$ & $190(64.0)$ & $212(93.7)$ & 0.07 & & $226(84.3)$ & $282(119)$ & 0.1 \\
\hline Laminectomy, $\mathrm{n}(\%)$ & $332(96.2)$ & $174(96.1)$ & $74(97.4)$ & $>0.99$ & & $70(95.9)$ & $14(93.3)$ & 0.53 \\
\hline Interbody graft placement, $\mathrm{n}(\%)$ & $304(88.1)$ & $152(84.0)$ & $75(98.7)$ & $\mathbf{0 . 0 0 1}$ & & $62(84.9)$ & $15(100)$ & 0.2 \\
\hline
\end{tabular}

$\mathrm{EBL}=$ estimated blood loss.

Boldface type indicates statistical significance. 
TABLE 4. Summary of postoperative outcomes

\begin{tabular}{|c|c|c|c|c|c|c|c|}
\hline \multirow[b]{2}{*}{ Variable } & \multirow[b]{2}{*}{ All $(n=345)$} & \multicolumn{3}{|c|}{ 1-Level Fusion } & \multicolumn{3}{|c|}{ 2-Level Fusion } \\
\hline & & Open $(n=181)$ & MIS $(n=76)$ & $p$ Value & Open $(n=73)$ & MIS (n = 15) & $\mathrm{p}$ Value \\
\hline LOS in days, mean (SD) & $3.46(1.66)$ & $3.36(1.55)$ & $3.21(1.74)$ & 0.53 & $3.88(1.67)$ & $4.00(2.00)$ & 0.83 \\
\hline Home routine & $283(82.3)$ & $151(83.9)$ & $67(88.2)$ & & $54(74.0)$ & $11(73.3)$ & \\
\hline Home w/ home health care services & $18(5.23)$ & $11(6.11)$ & $3(3.95)$ & & $3(4.11)$ & $1(6.67)$ & \\
\hline 90-day readmission, $\mathrm{n}(\%)$ & $5(1.47)$ & $1(0.56)$ & $2(2.67)$ & 0.21 & $1(1.39)$ & $1(6.67)$ & 0.32 \\
\hline 90-day return to work, $\mathrm{n}(\%)$ & $87(66.4)$ & $42(60.9)$ & $26(76.5)$ & 0.18 & $15(62.5)$ & $4(100)$ & 0.27 \\
\hline Return to the op room w/in $1 \mathrm{yr}, \mathrm{n}(\%)^{*}$ & $20(5.81)$ & $9(5.00)$ & $4(5.26)$ & $>0.99$ & $5(6.85)$ & $2(13.3)$ & 0.34 \\
\hline \multicolumn{8}{|l|}{ 12-mo functional outcomes } \\
\hline NASS satisfaction, $n(\%)$ & & & & 0.16 & & & 0.50 \\
\hline 4 & $20(6.85)$ & $9(6.00)$ & $1(1.56)$ & & $8(13.8)$ & $2(14.3)$ & \\
\hline Change in ODI, mean (SD) & $-24.17(17.4)$ & $-25.54(16.9)$ & $-27.61(16.4)$ & 0.40 & $-15.89(16.0)$ & $-27.43(23.2)$ & 0.1 \\
\hline Change in EQ-5D, mean (SD) & $0.24(0.22)$ & $0.25(0.22)$ & $0.26(0.21)$ & 0.84 & $0.15(0.24)$ & $0.27(0.20)$ & 0.08 \\
\hline Change in NRS-BP, mean (SD) & $-3.79(3.11)$ & $-4.22(2.96)$ & $-3.80(3.12)$ & 0.35 & $-2.68(3.23)$ & $-3.50(3.28)$ & 0.41 \\
\hline Change in NRS-LP, mean (SD) & $-4.00(3.48)$ & $-4.15(3.57)$ & $-4.47(3.11)$ & 0.51 & $-2.84(3.60)$ & $-4.93(2.70)$ & 0.02 \\
\hline Quality-adjusted life days w/in the 1st yr & $266(46.3)$ & $268(46.3)$ & $270(46.3)$ & 0.84 & $253(49.0)$ & $262(25.8)$ & 0.45 \\
\hline
\end{tabular}

* Related to the index procedure.
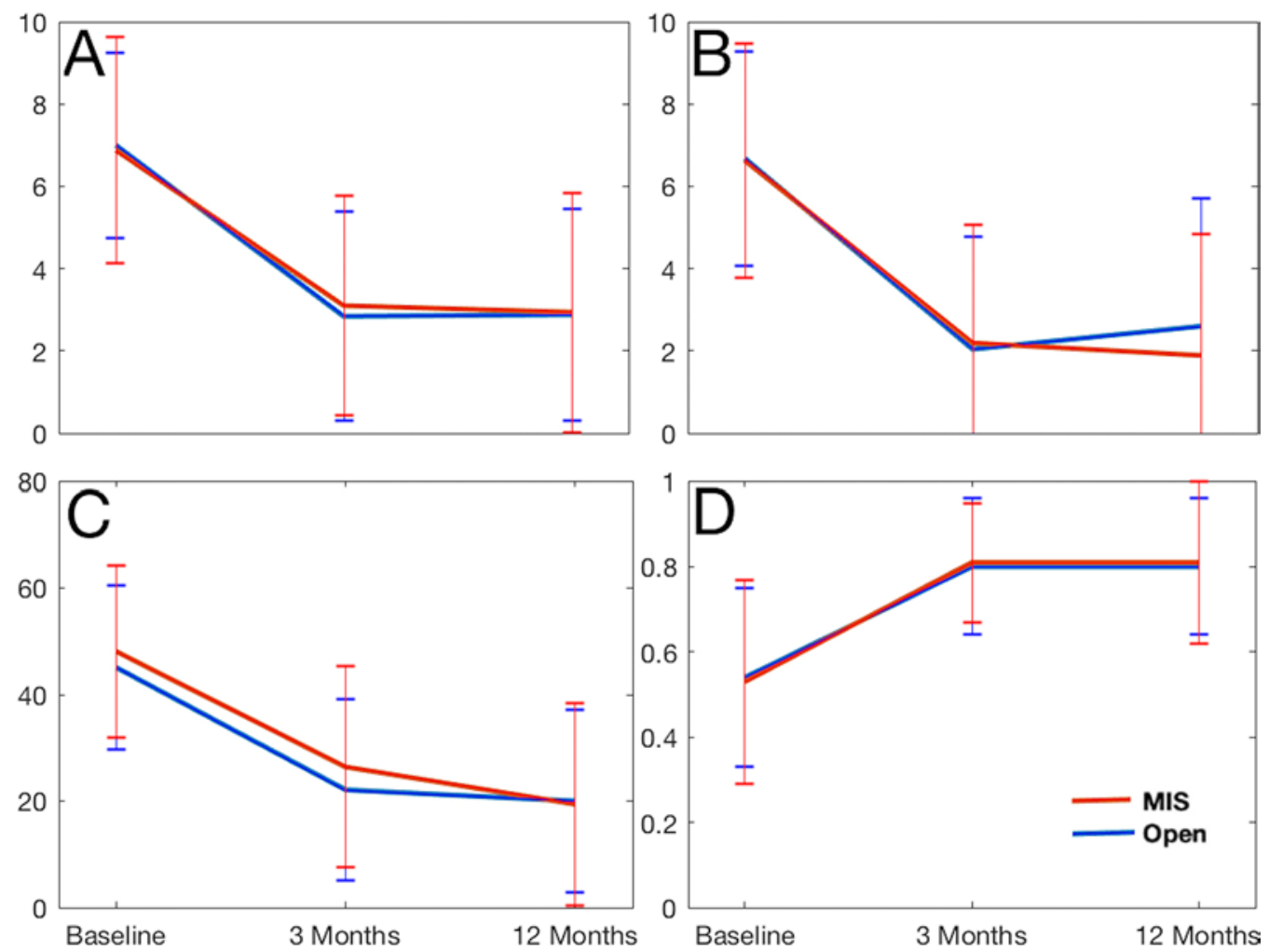

FIG. 2. PROs for patients undergoing 1-level fusion were collected at baseline, 3-month, and 12-month time periods. At baseline, all PRO scores were similar between the groups. Each group improved significantly from baseline at 3-and 12-month time periods in terms of NRS-BP (A), NRS-BP (B), ODI (C), and EQ-5D (D) scores ( $p<0.001$ [for each]). There were no significant differences between 3 months and 12 months between the 2 groups in terms of NRS-BP, NRS-LP, ODI, and EQ-5D scores. 

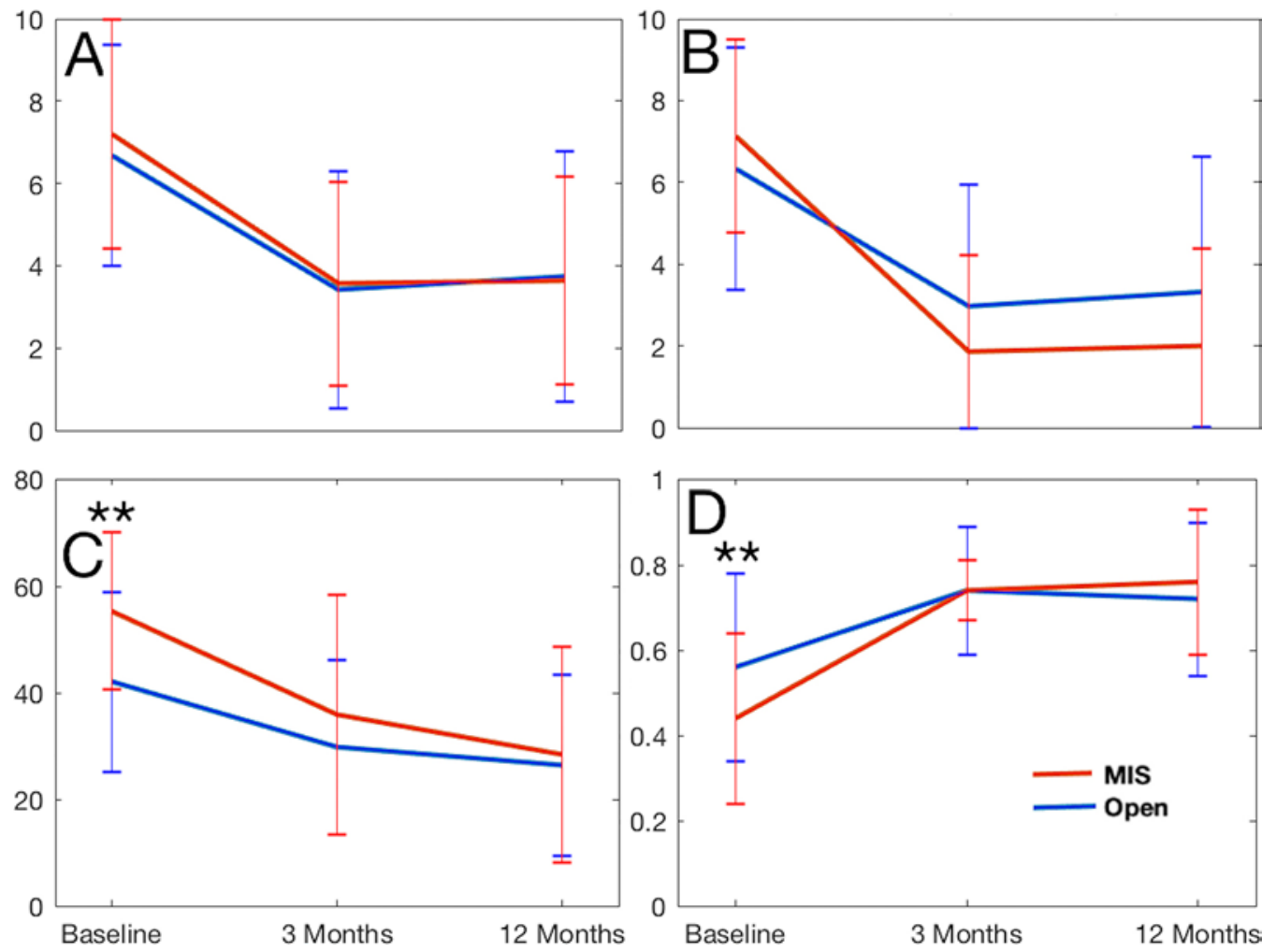

FIG. 3. PROs for patients undergoing 2-level fusion were collected at baseline, 3-month, and 12-month time periods. Each group improved significantly from baseline at the 3- and 12-month time periods in terms of NRS-BP (A), NRS-LP (B), ODI (C), and EQ-5D (D) scores $(p<0.001$ [for each]). However, at baseline, ODI and EQ-5D scores were significantly worse in the MIS group $(p=0.006$ and $p=0.049$, respectively). There were no significant differences between 3 months and 12 months between the groups in terms of NRS$\mathrm{BP}, \mathrm{NRS}-\mathrm{LP}, \mathrm{ODI}$, and EQ-5D scores. The asterisks represent significantly different scores. The lines represent means with SDs.

tive spine disease, including spondylolisthesis, is steadily increasing among spinal surgeons. ${ }^{6}$ While there are many potential benefits to the MIS approach, there is still a paucity of strong Class I evidence proving its efficacy and safety over conventional open approaches for the management of spondylolisthesis. In the present study, we analyzed data from 345 patients enrolled in a prospective, national spine registry who were treated by both orthopedic and neurological surgeons at 11 institutions across the United States. After using robust statistical methodology, we found that both approaches are very effective in treating the underlying pathology and restoring back and leg pain and function. Overall, postoperative functional and surgical outcomes were comparable between the MIS and open surgery cohorts.

A recently published systematic review and meta-analysis of 10 studies including 602 patients revealed that there was no significant difference in terms of functional or pain outcomes (ODI and visual analog scale, respectively), between open surgery and MIS. ${ }^{15}$ In the present study, patients in both groups presented with similar baseline comorbidity burdens and disease severity, thus minimizing the risk for selection bias in favor of the MIS approach. The impact of the MIS approach was not found to be significant for 12-month PROs, a finding that might be attributed to the limited sample size. However, a greater change in NRS-LP ( 2 points) was noted in the 2-level fusion subgroup. Interestingly, although not statistically significant, a higher proportion of patients in the MIS group reported that they were satisfied with the procedure (1-level: $91 \%$ vs $85 \%$; 2 levels: $79 \%$ vs $74 \%$ ), reflecting its promising value for treating this patient population.

Authors have previously shown that MIS is associated with less intraoperative blood loss and shorter hospital LOS. ${ }^{15}$ Similarly, in our study, blood loss in the MIS group was more than half of that recorded in the open group at the expense of slightly longer operative times (an average of 32 minutes for 1-level and 56 minutes for 2-level fusions), which is anticipated given that MIS requires deep knowledge of the relevant anatomy and comfort with the novel instrumentation and restricted view. ${ }^{13,15}$ We did not

TABLE 5. Adjusted odds ratios of approach on outcomes for patients undergoing 1-level fusion

\begin{tabular}{lccc}
\hline \multicolumn{1}{c}{ Outcome } & OR & $95 \% \mathrm{Cl}$ & $\mathrm{p}$ Value \\
\hline 12-mo ODI & 1.01 & $0.59-1.72$ & 0.96 \\
\hline 12-mo EQ5D & 0.87 & $0.52-1.48$ & 0.61 \\
\hline 12-mo NRS-BP & 1.05 & $0.62-1.78$ & 0.85 \\
\hline 12-mo NRS-LP & 0.69 & $0.39-1.21$ & 0.20 \\
\hline 12-mo patient satisfaction & 1.52 & $0.75-3.10$ & 0.25 \\
\hline LOS & 0.80 & $0.46-1.39$ & 0.43 \\
\hline 90-day return to work & 1.46 & $0.21-10.3$ & 0.70 \\
\hline
\end{tabular}

Reference group is the open surgery group. 
observe a difference with regard to postoperative recovery; patients in both cohorts spent approximately 3-4 days in the hospital, which may attributed to the lower LOS in the open group than that reported in previous studies. ${ }^{2,3,5,9,19,20}$

Finally, the rates for observed readmission and return to the operating room were very low in both groups; the second largest study to date by Parker and colleagues investigated 100 patients (50 in each arm) who underwent transforaminal interbody fusion (TLIF) for spondylolisthesis. ${ }^{19}$ The authors found similar surgical morbidity and hospital readmission rates for MIS and open TLIF. Also, although we did not detect a statistically significant difference, patients in the MIS group were over 15\% more likely to return to work within 90 days. Similarly, in the study by Parker et al., patients undergoing minimally invasive TLIF returned to work faster by a median of 4 weeks (7 vs 11 weeks). Overall, the findings of the present study confirm that the MIS approach represents a viable and safe alternative in the management of spondylolisthesis, involving less surgical trauma while achieving similar functional outcomes to open surgery.

\section{Limitations}

This study has several limitations. To start, this is a nonrandomized study; therefore, there is a risk for selection bias during preoperative planning, and the type of surgical procedure to be performed is at the surgeon's discretion. We are also limited by the 12-month follow-up duration and cannot provide insight into associated PROs, complications, and reoperations beyond that time frame. Furthermore, our study lacks radiographic data, including follow-up radiographs. Lastly, there was moderate heterogeneity of the MIS practices, including the utilization of tubular and percutaneous screw fixation.

\section{Conclusions}

The present study evaluated real-world spinal practice for patients undergoing open or minimally invasive lumbar fusion for Grade I degenerative spondylolisthesis. Both approaches were found to be effective in addressing patients' symptoms and disability. Overall, no difference was detected with regard to 12-month PROs, LOS, and 90-day return to work; however, larger changes in functional outcome scores were observed in the 2-level fusion subgroup. Future studies are needed to provide insight into the long-term efficacy and cost-effectiveness of the 2 treatment modalities.

\section{Acknowledgments}

We would like to thank all of the site research coordinators for their help with data extraction and validation. We would also like to thank the Neurosurgery Research and Education Foundation for its financial support of this work.

\section{References}

1. Andersson GB: Epidemiological features of chronic low-back pain. Lancet 354:581-585, 1999

2. Archavlis E, Carvi y Nievas M: Comparison of minimally invasive fusion and instrumentation versus open surgery for severe stenotic spondylolisthesis with high-grade facet joint osteoarthritis. Eur Spine J 22:1731-1740, 2013
3. Cheng JS, Park P, Le H, Reisner L, Chou D, Mummaneni PV: Short-term and long-term outcomes of minimally invasive and open transforaminal lumbar interbody fusions: is there a difference? Neurosurg Focus 35(2):E6, 2013

4. Daltroy LH, Cats-Baril WL, Katz JN, Fossel AH, Liang MH: The North American Spine Society lumbar spine outcome assessment instrument: reliability and validity tests. Spine (Phila Pa 1976) 21:741-749, 1996

5. Dhall SS, Wang MY, Mummaneni PV: Clinical and radiographic comparison of mini-open transforaminal lumbar interbody fusion with open transforaminal lumbar interbody fusion in 42 patients with long-term follow-up. J Neurosurg Spine 9:560-565, 2008

6. Dyrda L: Minimally invasive vs. open spine surgery: 6 key points. Becker's Spine Review. January 30, 2014. (http:// www.beckersspine.com/spine/item/19204-minimallyinvasive-vs-open-spine-surgery-6-key-points) [Accessed June 12, 2017]

7. EuroQol Group: EuroQol--a new facility for the measurement of health-related quality of life. Health Policy 16:199208, 1990

8. Fairbank JC, Pynsent PB: The Oswestry Disability Index. Spine (Phila Pa 1976) 25:2940-2952, 2000

9. Ghahreman A, Ferch RD, Rao PJ, Bogduk N: Minimal access versus open posterior lumbar interbody fusion in the treatment of spondylolisthesis. Neurosurgery 66:296-304, 2010

10. Harris EB, Sayadipour A, Massey P, Duplantier NL, Anderson DG: Mini-open versus open decompression and fusion for lumbar degenerative spondylolisthesis with stenosis. Am J Orthop 40:E257-E261, 2011

11. Kalichman L, Kim DH, Li L, Guermazi A, Berkin V, Hunter DJ: Spondylolysis and spondylolisthesis: prevalence and association with low back pain in the adult community-based population. Spine (Phila Pa 1976) 34:199-205, 2009

12. Khan NR, Clark AJ, Lee SL, Venable GT, Rossi NB, Foley KT: Surgical outcomes for minimally invasive vs open transforaminal lumbar interbody fusion: an updated systematic review and meta-analysis. Neurosurgery 77(6):847-874, 2015

13. Kotani Y, Abumi K, Ito M, Sudo H, Abe Y, Minami A: Midterm clinical results of minimally invasive decompression and posterolateral fusion with percutaneous pedicle screws versus conventional approach for degenerative spondylolisthesis with spinal stenosis. Eur Spine J 21:1171-1177, 2012

14. Langley GB, Sheppeard H: The visual analogue scale: its use in pain measurement. Rheumatol Int 5:145-148, 1985

15. Lu VM, Kerezoudis P, Gilder HE, McCutcheon BA, Phan K, Bydon M: Minimally invasive surgery versus open surgery spinal fusion for spondylolisthesis: a systematic review and meta-analysis. Spine (Phila Pa 1976) 42:E177-E185, 2017

16. McGirt MJ, Speroff T, Dittus RS, Harrell FE Jr, Asher AL: The National Neurosurgery Quality and Outcomes Database $\left(\mathrm{N}^{2} \mathrm{QOD}\right)$ : general overview and pilot-year project description. Neurosurg Focus 34(1):E6, 2013

17. Meyerding HW: Diagnosis and roentgenologic evidence in spondylolisthesis. Radiology 20:108-120, 1933

18. Park Y, Ha JW: Comparison of one-level posterior lumbar interbody fusion performed with a minimally invasive approach or a traditional open approach. Spine (Phila Pa 1976) 32:537-543, 2007

19. Parker SL, Mendenhall SK, Shau DN, Zuckerman SL, Godil SS, Cheng JS, et al: Minimally invasive versus open transforaminal lumbar interbody fusion for degenerative spondylolisthesis: comparative effectiveness and cost-utility analysis. World Neurosurg 82:230-238, 2014

20. Rampersaud YR, Gray R, Lewis SJ, Massicotte EM, Fehlings MG: Cost-utility analysis of posterior minimally invasive fusion compared with conventional open fusion for lumbar spondylolisthesis. SAS J 5:29-35, 2011 
21. Sedney CL, McConda DB, Daffner SD: Natural history of spondylolysis and spondylolisthesis. Semin Spine Surg 26:214-218, 2014

22. Sulaiman WAR, Singh M: Minimally invasive versus open transforaminal lumbar interbody fusion for degenerative spondylolisthesis grades 1-2: patient-reported clinical outcomes and cost-utility analysis. Ochsner J 14:32-37, 2014

23. Tsutsumimoto T, Shimogata M, Ohta H, Misawa H: Miniopen versus conventional open posterior lumbar interbody fusion for the treatment of lumbar degenerative spondylolisthesis: comparison of paraspinal muscle damage and slip reduction. Spine (Phila Pa 1976) 34:1923-1928, 2009

24. Wang J, Zhou Y, Zhang ZF, Li CQ, Zheng WJ, Liu J: Comparison of one-level minimally invasive and open transforaminal lumbar interbody fusion in degenerative and isthmic spondylolisthesis grades 1 and 2. Eur Spine J 19:1780-1784, 2010

\section{Disclosures}

The authors report the following. Dr. Mummaneni: consultant for DePuy Spine and Stryker; direct stock ownership in Spinicity/ ISD; clinical/research support for this study from NREF; royalties from DePuy Spine, Thieme Publishers, and Springer Publishers; grant from AOSpine; and honoraria from Globus. Bisson: consultant for nView. Glassman: employee of Norton Healthcare; patent holder with, consultant for, and royalties from Medtronic; NuVasive (provides funds directly to the database company; no funds are paid directly to individual or institution); and past president of the Scoliosis Research Society. Foley: consultant for, direct stock ownership in, and patent holder with Medtronic; direct stock ownership in NuVasive; and direct stock ownership in Spine Wave. Slotkin: consultant for Stryker Spine. CI Shaffrey: direct stock ownership in, consultant for, and patent holder with NuVasive; consultant for Zimmer Biomet; patent holder with
Medtronic; and patent holder with Zimmer Biomet. Coric: consultant for and direct stock ownership in Spine Wave; consultant for Stryker; consultant for Medtronic; consultant for and direct stock ownership in Premia Spine; and direct stock ownership in Spinal Kinetics. Knightly: NPA board of directors. Park: consultant for and royalties from Globus; consultant for NuVasive; consultant for Zimmer Biomet; and consultant for Medtronic. Fu: consultant for SI-BONE. Devin: consultant for and clinical/research support for this study from Stryker Spine; and consultant for Pacira.

\section{Author Contributions}

Conception and design: Bydon, Mummaneni, Bisson, Glassman, Foley, Slotkin, Park. Acquisition of data: Bydon, Mummaneni, Bisson, Kerezoudis, Glassman, Foley, Slotkin, Potts, M Shaffrey, CI Shaffrey, Coric, Knightly, Devin, Chotai. Analysis and interpretation of data: Bydon, Mummaneni, Bisson, Kerezoudis, Glassman, Foley, Slotkin, Potts, M Shaffrey, CI Shaffrey, Coric, Knightly, Devin, Chotai, Virk. Drafting the article: Bydon, Mummaneni, Bisson, Kerezoudis, Glassman, Foley, Slotkin, Potts, M Shaffrey, CI Shaffrey, Coric, Knightly, Fu, Devin, Chotai, Chan, Virk. Critically revising the article: Bydon, Mummaneni, Bisson, Glassman, Foley, Slotkin, Potts, M Shaffrey, CI Shaffrey, Coric, Knightly, Park, Fu, Devin, Chotai, Chan, Virk, Asher. Reviewed submitted version of manuscript: all authors. Approved the final version of the manuscript on behalf of all authors: Bydon. Statistical analysis: Bydon, Mummaneni, Bisson, Glassman, Foley, Slotkin, Potts, M Shaffrey, CI Shaffrey, Coric, Knightly, Park, Fu, Chan, Virk, Asher. Administrative/technical/material support: Mummaneni, Foley, Slotkin, Knightly, Park, Asher. Study supervision: Mummaneni, Asher.

\section{Correspondence}

Mohamad Bydon, Department of Neurosurgery, Mayo Clinic, 200 First St. SW, Rochester, MN 55905. email: bydon.mohamad@ mayo.edu. 